\title{
THE COMPLETION OF PRÜFER DOMAINS
}

\author{
JIŘí MOČKOř
}

\begin{abstract}
The completion of Prüfer and almost Dedekind domains in topologies $\sup \left\{\mathscr{T}_{w}: w \in \Omega\right\}$, where $\mathscr{T}_{w}$ is a topology induced by a valuation $w \in \Omega$, are characterized in terms of Manis valuations.
\end{abstract}

1. Let $A$ be an integral domain with the quotient field $K$ and let $\Omega$ be the family of nontrivial valuations on $K$ which are nonnegative on $A$. It is well known that a valuation $w \in \Omega$ with the value group $G_{w}$ defines a field topology $\mathscr{T}_{w}$ in $K$ with the sets $U_{w, \alpha}=\{x \in K: w(x)>\alpha\}, \alpha \in G_{w}^{+}=\{\beta$ $\left.\in G_{w}: \beta \geqslant 0\right\}$, as a base of the neighbourhoods of zero in $K$. Since $\widetilde{T}_{w}$ is a minimal topology in the ordered set of all field topologies on $K$, the completion $\hat{K}_{w}$ of $K$ is a field and the extension $\hat{w}$ of $w$ on $\hat{K}_{w}$ is a valuation on $\hat{K}_{w}$.

In this note we consider a more general situation. Let $\mathcal{T}$ be the supremum of the family of topologies $\left\{\mathcal{T}_{w}: w \in \Omega\right\}$. In general, the family $\Omega$ contains nonequivalent valuations, and, in this case, the completion $\hat{K}$ of $K$ in $\mathcal{\sigma}$ may contain zero divisors. Hence, it seems natural to use for the investigation of the ideal-theoretic properties of the completion $\hat{A}$ of a domain $A$ in a topology $\mathcal{T}$ the valuation theory on rings with zero divisors which was introduced by M. E. Manis [8]. Especially if $A$ is a Prüfer domain or almost Dedekind domain, we determine to what extent these properties hold for $\hat{A}$.

In this paper all rings and groups are assumed to be commutative. At first, we recall some results of Manis [8] and M. Griffin [4]. A valuation on a ring $R$ is a map $w$ from $R$ onto a totally ordered group $G$ and a symbol $\infty$, such that for all $a, b \in R$,

(i) $w(a b)=w(a)+w(b)$,

(ii) $w(a+b) \geqslant \min \{w(a), w(b)\}$.

Note that in (ii) equality holds if $w(a) \neq w(b)$. The valuation ring $R_{w}$ of $w$ is defined to be the subring of $R$ of all elements with nonnegative value. The set $M(w)$ of all elements of $R$ with positive value is a prime ideal of $R_{w}$ and $\left(R_{w}, M(w)\right)$ is said to be a valuation pair associated with $w$. An ideal $Q$ of a valuation ring $R_{w}$ is $w$-closed if $x \in Q, y \in R_{w}$, and $w(x) \leqslant w(y)$ imply $y \in Q$. M. Griffin [4] defines the large quotient ring of a ring $R$ with respect to a multiplicative system $S$ of $R$ as $R_{[S]}=\{x \in K: x s \in R$ for some $s \in S\}$, where $K$ is the total quotient ring of $R$. If $P$ is a prime ideal of $R$ with

Received by the editors May 12, 1976 and, in revised form, April 25, 1977.

AMS (MOS) subject classifications (1970). Primary 13A15, $13 \mathrm{~J} 10$. 
$S=R-P, R_{[P]}$ will be used for $R_{[S]}$. Also $[P] R_{[P]}$ denotes the prime ideal of $R_{[P]}$ defined by $\{x \in K: x s \in P$ for some $s \in R-P\}$. Then a Prüfer ring is a ring in which every finitely generated regular ideal is invertible. By [4, Theorem 13], $R$ is a Prüfer ring if and only if for every maximal regular ideal $Q$ of $R,\left(R_{[Q]},[Q] R_{[Q]}\right)$ is a valuation pair associated with some valuation on $K$.

M. D. Larsen [7] introduced the notion of an $N$-ring which generalizes almost Dedekind domain, where an almost Dedekind domain is an integral domain $A$ such that for all prime ideals $P$ of $A, A_{P}$ is a discrete rank one valuation ring. He defines an $N$-ring to be a ring $R$ in which for all maximal regular prime ideals $Q$ of $R,\left(R_{[Q]},[Q] R_{[Q]}\right)$ is a valuation pair associated with a valuation $w$ such that $G_{w}$ is isomorphic to the group $Z$ of integers.

The author is grateful to the referee, especially for his suggestions concerning $h$-local Prüfer domains.

2. We begin this section with some results about the topology induced by a Manis valuation. Let $\left(R_{w}, M(w)\right)$ be a valuation pair and let $K$ be the total quotient ring of $R_{w}$. Then the sets

$$
U_{w, \alpha}=\{x \in K: w(x)>\alpha\}, \quad \alpha \in G_{w}^{+},
$$

form a base of the neighbourhoods of zero in $K$ for some ring topology $\mathcal{T}_{w}$ in $K$. For, $U_{w, \alpha}$ is an additive subgroup of $K, U_{w, \alpha} \cdot U_{w, \alpha} \subseteq U_{w, \alpha}$, and for any $x_{0} \in K, \alpha \in G_{w}^{+}$, we have $x_{0} U_{w, \beta} \subseteq U_{w, \alpha}$, where $\beta \geqslant \alpha-w\left(x_{0}\right)$ if $x_{0} \notin$ $w^{-1}(\infty)$ and $\beta \in G_{w}^{+}$for $x_{0} \in w^{-1}(\infty)$. Now, if we define on the set $G_{w}^{\prime}=G_{w} \cup\{\infty\}$ a topology by setting $\bar{X}=X \cup\{\infty\}$ for every $X \subseteq G_{w}^{\prime}$, $X \neq \varnothing, \bar{\varnothing}=\varnothing$, then $G_{w}^{\prime}$ is a complete uniform space and $w:\left(K, \mathcal{J}_{w}\right) \rightarrow G_{w}^{\prime}$ is continuous.

LEMMA 1. Let $R_{w}$ be a valuation ring with $J$ an ideal of $R_{w}$. If $J$ is $w$-closed and $J \neq w^{-1}(\infty)$, it is clopen in $R_{w}$. If $J$ is closed, then $w^{-1}(\infty) \subseteq J$.

Proof. Let $J$ be $w$-closed Since $J \neq w^{-1}(\infty)$, there exists $a \in J-w^{-1}(\infty)$. Then for every $x \in J$ and $y \in U_{w, w(a)}$ we have either $w(x+y) \geqslant w(x)$ or $w(x+y) \geqslant w(a)$, hence $x+U_{w, w(a)} \subseteq J$ and $J$ is open in $R_{w}$. Since $J$ is a subgroup of $R_{w}, J$ is clopen. Now let $J$ be closed in $R_{w}$ and let $x \in w^{-1}(\infty)$. Then $x \in \overline{\{0\}}$, the closure of $\{0\}$ in $R_{w}$, and $\overline{\{0\}} \subseteq J$.

LeMma 2. A ring $K$ contains no zero divisors if there exists a nontrivial valuation on $K$ such that the associated topology is Hausdorff.

Proof. Suppose that there exists a nontrivial valuation $w$ on $K$ such that $\left(K, \mathscr{T}_{w}\right)$ is Hausdorff. Then $w^{-1}(\infty) \subseteq \overline{\{0\}}=\{0\}$. If there is a zero divisor in $K$, then there exists $x \in w^{-1}(\infty), x \neq 0$, which cannot happen.

Further, suppose that $A$ is an integral domain with the quotient field $K$ and let $\Omega$ be the family of nontrivial valuations on $K$ which are nonnegative on $A$ and put

$$
\mathscr{T}=\sup \left\{\mathcal{T}_{w}: w \in \Omega\right\}
$$


If $\left(\hat{K}_{w}, \hat{\mathcal{T}}_{w}\right)$ and $(\hat{K}, \hat{\mathcal{T}})$ are the completions of $\left(K, \mathcal{T}_{w}\right)$ and $(K, \mathcal{T})$, respectively, we denote by $\hat{w}, \tilde{w}$ the continuous extension of $w$ on $\hat{K}_{w}$ and $\hat{K}$, respectively. It is well known that $\hat{w}$ is a valuation on the field $\hat{K}_{w}$ and $\hat{\sigma}_{w}=\sigma_{\hat{w}}$.

LEMMA 3. $\tilde{w}$ is a Manis valuation on $\hat{K}$ for any $w \in \Omega$.

Proof. We obtain the result from the fact that $K$ is a dense subset in the Hausdorff space $\hat{K}$ and $\tilde{w}$ is continuous.

Now, since $\tilde{w}$ is a Manis valuation on $\hat{K}$, it defines a topology $\mathscr{T}_{\bar{w}}$ on $\hat{K}$. Then we can prove the following:

Proposition 4. $\hat{\mathcal{J}}=\sup \left\{\mathscr{T}_{\tilde{w}}: w \in \Omega\right\}$.

Proof. We consider the following diagram

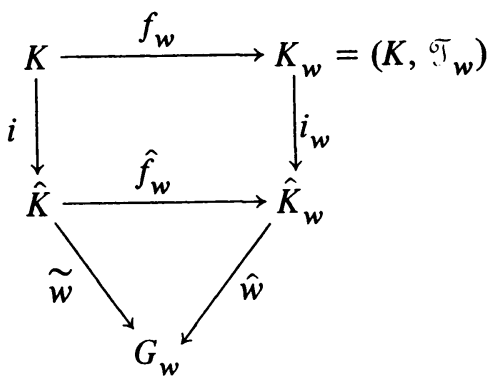

where $w \in \Omega, \hat{f}_{w}$ is the continuous extension of $f_{w}=\mathrm{id}_{K}$ and $i, i_{w}$ are the canonical injections. We have $\tilde{w} \cdot i=w, \hat{f}_{w} \cdot i=i_{w} \cdot f_{w}, w=\hat{w} \cdot i_{w} \cdot f_{w}$; thus $\tilde{w} \cdot i=\hat{w} \cdot \hat{f}_{w} \cdot i$. Since $i(K)$ is a dense subset in the Hausdorff space $\hat{K}$, we obtain $\tilde{w}=\hat{w} \cdot \hat{f}_{w}$. Now, since $U_{\tilde{w}, \alpha}=\hat{f}_{w}^{-1}\left(U_{\hat{w}, \alpha}\right)$ for every $\alpha \in G_{w}^{+}, w \in \Omega$, $U_{\hat{\boldsymbol{w}}, \alpha}$ is open in $\hat{K}_{w}$; it follows that $U_{\tilde{\boldsymbol{w}}, \alpha}$ is open in $\hat{\mathfrak{T}}$; thus $\hat{\mathfrak{T}} \geqslant \mathcal{T}_{\tilde{w}}$ for every $w \in \Omega$. Suppose that $\mathcal{G}$ is a topology on $\hat{K}$ such that $\mathcal{G} \geqslant \mathcal{T}_{\bar{w}}$ for every $w \in \Omega$. Then from the fact that $\tilde{w}=\hat{w} \cdot \hat{f}_{w}$, we obtain that $\hat{f}_{w}$ is continuous in $(\hat{K}, \mathcal{G})$ for every $w \in \Omega$. Thus, using the fact $\mathcal{T}=\sup \left\{\mathcal{T}_{w}: w \in \Omega\right\}$, we obtain by [2, $\S 3$, Proposition 18], that the topology $\hat{\mathcal{G}}$ is the supremum of topologies on $\hat{K}$ for which $\hat{f}_{w}, w \in \Omega$, are continuous. Hence, $\hat{\mathcal{T}} \leqslant \mathcal{G}$ and $\hat{\mathcal{G}}=\sup \left\{\mathcal{T}_{\tilde{w}}\right.$ : $w \in \Omega\}$.

Henceforth, we assume that $K$ is a subring of $\hat{K}$.

Proposition 5. Let $w \in \Omega$ and let $\left(R_{\tilde{w}}, M(\tilde{w})\right)$ be the valuation pair associated with $\tilde{w}$. Let $R_{w}$ be a valuation ring with the maximal ideal $M(w)$ of a valuation $w$ in a field $K$. Then

$$
R_{\tilde{w}}=\overline{R_{w}}, \quad M(\tilde{w})=\overline{M(w)},
$$

where $\bar{X}$ is the closure of $X$ in $(\hat{K}, \hat{\jmath})$.

Proof. Let $x \in \hat{K}-\overline{R_{w}}$. Then by Proposition 4 , there exist $w_{1}, \ldots, w_{n} \in$ $\Omega, \alpha_{i} \in G_{w_{i}}^{+}, i=1, \ldots, n$, such that 


$$
\left(x+\bigcap_{i=1}^{n} U_{\tilde{x}_{i}, \alpha_{i}}\right) \cap R_{w}=\varnothing .
$$

There is no loss of generality in assuming that $w \in\left\{w_{1}, \ldots, w_{n}\right\}$, say $w=w_{1}$. Moreover, since $K$ is a dense subset in $\hat{K}$, there exists $z \in K$ such that

$$
\tilde{w}_{i}(z-x)>\alpha_{i}, \quad i=1, \ldots, n .
$$

Hence, $z \in K-R_{w}$ and $w(z)=w_{1}(z)<0$. Suppose that $w_{1}(z) \neq \tilde{w}_{1}(x)$. Then

$$
\alpha_{1}<\tilde{w}_{1}(z-x)=\min \left\{\tilde{w}_{1}(x), w_{1}(z)\right\}<0,
$$

a contradiction with $\alpha_{1} \geqslant 0$. Thus, $\tilde{w}(x)=w(z)<0$ and $x \notin R_{\tilde{w}}$. Therefore, $R_{\tilde{w}} \subseteq \overline{R_{w}}$. On the other hand, $R_{\tilde{w}}$ is closed in $\hat{K}$ and we have $\overline{R_{w}} \subseteq R_{\tilde{w}}$. Thus, $R_{\bar{w}}=\overline{R_{w}}$. The rest can be proved analogously.

For future investigation we need to solve the following problem. If $w$ is a valuation on the quotient field $K$ of $A$ such that $R_{w}=A_{P(w)}$, where $P(w)=$ $M(w) \cap A$, when $R_{\hat{w}}=\left(\hat{A}_{w}\right)_{P(\hat{w})}$ holds, where $\hat{A}_{w}$ is the closure of $A$ in $\hat{K}_{w}$ and $P(\hat{w})=M(\hat{w}) \cap \hat{A}_{w}$. The following example shows that it does not hold in general.

ExAmple. Let $Q$ be the field of rational numbers and let $X$ be an indeterminate over $Q$. In the field $Q(X)$ we define a valuation $w$ in the following way.

$$
\begin{gathered}
w\left(\sum_{i=0}^{n} a_{i} X^{i}\right)=k \Leftrightarrow a_{0}=\cdots=a_{k-1}=0, \quad a_{k} \neq 0 ; \\
w(f / g)=w(f)-w(g), \quad f, g \in Q[X], g \neq 0, w(0)=\infty .
\end{gathered}
$$

Then $R_{w}$ is a discrete rank one valuation ring and $R_{w}=Z[X]_{M(w) \cap Z[X]}$. Now in the quotient field $Q((X))$ of the ring $Q[[X]]$ of formal power series over $Q$, we define a valuation $\hat{w}$ in the following way.

$$
\begin{gathered}
\hat{w}\left(\sum_{n=0}^{\infty} a_{n} X^{n}\right)=k \Leftrightarrow a_{0}=\cdots=a_{k-1}=0, \quad a_{k} \neq 0, a_{n} \in Q ; \\
\hat{w}(f / g)=\hat{w}(f)-\hat{w}(g), \quad f, g \in Q[[X]], g \neq 0, \hat{w}(0)=\infty .
\end{gathered}
$$

Then $\left(Q((X)), \mathcal{T}_{\hat{w}}\right)$ is the completion of $\left(Q(X), \mathscr{T}_{w}\right), \hat{w}$ is the unique continuous extension of $w$ on $Q((X))$ and $Z[[X]]$ is the closure of $Z[X]$ in $Q((X))$. To show that $R_{\hat{w}} \neq Z[[X]]_{M(\hat{w}) \cap Z[[X]]}$, we need a simple lemma.

Lemma. Let $n$ be a natural number, $z_{i} \in Z^{+}, i=0, \ldots, n-1$, be such that $0 \leqslant z_{i}<p_{n-i}$, where $p_{j}$ is the jth prime number and let $z_{0}\left(1 / p_{n}\right)+z_{1}\left(1 / p_{n-1}\right)$ $+\cdots+z_{n-1}(1 / 2) \in Z$. Then $z_{0}=z_{1}=\cdots=z_{n-1}=0$.

Proof. The proof is by induction on $n$. The lemma holds for $n=1$. We assume that the lemma holds for $n$. Let $z_{i} \in Z, i=0, \ldots, n$, be such that $0 \leqslant z_{i}<p_{n+1-i}$, and let $z_{0}\left(1 / p_{n+1}\right)+\cdots+z_{n}(1 / 2) \in Z$. Let $z_{i}^{\prime}, \quad i=$ 
$0, \ldots, n-1$, be such that

$$
z_{i+1} p_{n+1}=z_{i}^{\prime} \quad\left(\bmod p_{n-i}\right), \quad 0 \leqslant z_{i}^{\prime}<p_{n-i} .
$$

Then $z_{0}^{\prime}\left(1 / p_{n}\right)+\cdots+z_{n-1}^{\prime}(1 / 2) \in Z$, and by induction we have $z_{0}^{\prime}$ $=\cdots=z_{n-1}^{\prime}=0$. Hence, $z_{1} p_{n+1} / p_{n} \in Z, \ldots, z_{n} p_{n+1} / 2 \in Z$, and we obtain $z_{0}=z_{1}=\cdots=z_{n}=0$.

Now, $f=1+(1 / 2) X+\cdots+\left(1 / p_{n}\right) X^{n}+\cdots \in R_{\hat{w}}$. We suppose that there exist $u=\sum a_{n} X^{n}, v=\sum b_{n} X^{n} \in Z[[X]]$ such that $a_{0} \neq 0$ and $v=f \cdot u$. Then for any natural $n$ we have

$$
b_{n}=a_{n}+(1 / 2) a_{n-1}+\cdots+\left(1 / p_{n}\right) a_{0} .
$$

Let $a_{n-i}=z_{n-i}\left(\bmod p_{i}\right), i=1, \ldots, n, \quad 0 \leqslant z_{n-i}<p_{i}$. Then $z_{0}\left(1 / p_{n}\right)+\cdots+z_{n-1}(1 / 2) \in Z$, and by the lemma we have $z_{0}=\cdots=$ $z_{n-1}=0$. Thus, $p_{n}$ divides $a_{0}$ for every $n$, a contradiction.

The partial solution of the problem gives the following two propositions.

Proposition 6. Let $w$ be a discrete rank one valuation on the quotient field $K$ of $A$ such that $R_{w}=A_{P(w)}$ and let $P(w)$ be a maximal ideal of $A$. Then $R_{\hat{w}}=\hat{A}_{w}$.

Proof. It is well known that in this case $\mathcal{T}_{w}$ is the $M(w)$-adic topology. Then $\hat{A}_{w}$ is the completion of $A$ in $P(w)=M(w) \cap A$-adic topology and, since $P(w)$ is maximal in $A, \hat{A_{w}}$ is local with the unique maximal ideal $P(\hat{w})=M(\hat{w}) \cap \hat{A}_{w}$. Hence, $R_{w}=A_{P(w)} \subseteq\left(\hat{A}_{w}\right)_{P(\hat{w})}=\hat{A}_{w}$ and $R_{\hat{w}}=\overline{R_{w}} \subseteq \hat{A}_{w}$ $\subseteq R_{\hat{w}}$, where the vinculum denotes the closure in $K_{w}$. Therefore, $R_{\hat{w}}=\hat{A}_{w}$.

Using the example it should be observed that " $P(w)$ be maximal" cannot be removed from Proposition 6.

The following proposition follows immediately from [10, Theorem 2.1].

Proposition 7. $R_{\hat{w}}=\left(\hat{A}_{w}\right)_{P(\hat{w})}$ if and only if there exists an order epimorphism $f$ from a group of divisibility $G\left(\hat{A}_{w}\right)$ of $\hat{A}_{w}$ onto $G_{w}$ such that $f \cdot v=\hat{w}$, where $v$ is the canonical map from $\hat{K}_{w}^{*}$ onto $G\left(\hat{A}_{w}\right)$.

Further, if $R_{\hat{w}}=\left(\hat{A}_{w}\right)_{P(\hat{w})}$ for a valuation $w$, it is natural to ask when $R_{\tilde{w}}=\hat{A}_{[P(\tilde{w})]}$ for $P(\tilde{w})=M(\tilde{w}) \cap \hat{A}$. To solve this problem, we prove two lemmas.

At first, on the family $\Omega$ we may define an equivalence relation

$$
w \equiv w^{\prime} \quad \text { if and only if } w, w^{\prime} \text { are dependent. }
$$

Let $\Omega_{0}$ be a family of representatives of the equivalence classes.

L Емма 8. Let for every $w \in \Omega_{0}, x_{w}$ be an element from $\hat{K}_{w}$. Then there exists $x \in \hat{K}$ such that $\hat{f}_{w}(x)=x_{w}$ for every $w \in \Omega_{0}$. Furthermore, if $x_{w} \neq 0$ for every $w \in \Omega_{0}, x$ is regular.

Proof. We set $\Delta=\Pi\left\{i_{w}: w \in \Omega_{0}\right\}$, where $i_{w}: K \rightarrow \hat{K}_{w}$ is the canonical map. Then using the approximation theorem for independent valuations, we obtain that 


$$
\Delta: K \rightarrow \Pi\left\{\hat{K}_{w}: w \in \Omega_{0}\right\}
$$

is a completion of the topological field $(K, \mathscr{T})$, and the canonical projection map $\operatorname{pr}_{w}$ of $\Pi\left\{\hat{K}_{w}: w \in \Omega_{0}\right\}$ onto $\hat{K}_{w}$ is the unique continuous homomorphism such that

$$
i_{w} \cdot f_{w}=\operatorname{pr}_{w} \cdot \Delta, \quad w \in \Omega_{0} .
$$

Now, since $i: K \rightarrow \hat{K}$ is the completion of $K$, there exists an isomorphism $\varphi$ such that $\varphi \cdot \Delta=i$. Then $\left(\hat{f}_{w} \cdot \varphi\right) \cdot \Delta=\hat{f}_{w} \cdot i=i_{w} \cdot f_{w}$, hence $\operatorname{pr}_{w}=\hat{f}_{w} \cdot \varphi$ for every $w \in \Omega_{0}$. Let $\left(x_{w}\right) \in \Pi\left\{\hat{K}_{w}: w \in \Omega_{0}\right\}$. We set $x=\varphi\left(\left(x_{w}\right)\right) \in \hat{K}$. Then $\hat{f}_{w}(x)=\operatorname{pr}_{w}\left(\left(x_{w}\right)\right)=x_{w}$ and if $x_{w} \neq 0$ for every $w \in \Omega_{0},\left(x_{w}\right)$ is regular in $\Pi\left\{\hat{K}_{w}: w \in \Omega_{0}\right\}$. Thus, $x$ is regular in $\hat{K}$.

For the second lemma we need some notation from [5]. Let $w, w^{\prime} \in \Omega$. If $R_{w} \subseteq R_{w^{\prime}}$ we say that $w^{\prime}$ is coarser than $w$ and write $w^{\prime} \leqslant w$. If $w^{\prime} \leqslant w$ then $G_{w^{\prime}} \cong G_{w} / H$, where $H$ is an isolated subgroup of $G_{w}$. Since the valuations coarser than $w$ are totally ordered there will be a finest valuation $w^{\prime \prime}=w^{\prime} \wedge$ $w$ coarser than both $w, w^{\prime}$. Let $H$ be a corresponding subgroup of $G_{w}$. Then we denote by ${ }_{\left(w, w^{\prime}\right)} \bar{\alpha}$ the element $\psi(\alpha)$, where $\psi: G_{w} \rightarrow G_{w^{\prime \prime}}$ is the canonical map and $\alpha \in G_{w}$. Now, let $w_{1}, \ldots, w_{n} \in \Omega$. Let $\left(\alpha_{1}, \ldots, \alpha_{n}\right) \in G_{w_{1}}$ $\times \cdots \times G_{w_{n}}, b_{i} \in K, 1 \leqslant i \leqslant n$. We define $\left(\left(\alpha_{1}, \ldots, \alpha_{n}\right),\left(b_{1}, \ldots, b_{n}\right)\right)$ to be concordant when the following conditions hold:

(1) if ${ }_{(i, j)} \bar{\alpha}_{i}={ }_{(j, i)} \bar{\alpha}_{j}$ then ${ }_{(i, j)} \bar{\alpha}_{i} \leqslant{ }_{(j, i)} \overline{w_{j}\left(b_{i}-b_{j}\right)}$;

(2) if ${ }_{(i, j)} \bar{\alpha}_{i}>_{(j, i)} \bar{\alpha}_{j}$ then ${ }_{(j, i)} \bar{\alpha}_{j}={ }_{(j, i)} w_{j}\left(b_{i}-b_{j}\right)$,

where ${ }_{(i, j)} \bar{\alpha}={ }_{\left(w_{i}, w_{j}\right)} \bar{\alpha}$. Then we say that $\Omega$ satisfies the weak reinforced approximation theorem (W.R.A.T.) for $A$ if, for any finite number $w_{i} \in \Omega$, $i=1, \ldots, n$, of valuations with $\left(\alpha_{1}, \ldots, \alpha_{n}\right) \in G_{w_{1}}^{+} \times \cdots \times G_{w_{n}}^{+}$and $\left(b_{1}, \ldots, b_{n}\right) \in A^{n}$ such that $\left(\left(\alpha_{1}, \ldots, \alpha_{n}\right),\left(b_{1}, \ldots, b_{n}\right)\right)$ is concordant, there exists $a \in A$ such that

$$
w_{i}\left(a-b_{i}\right)=\alpha_{i}, \quad i=1, \ldots, n .
$$

By [5, Proposition 24], for every Prüfer domain $A, \Omega$ satisfies the W.R.A.T.

LEMMA 9. Let $\Omega$ satisfy the W.R.A.T. for A. Then

$$
\hat{A}=\bigcap\left\{\hat{f}_{w}^{-1}\left(\hat{A}_{w}\right): w \in \Omega_{0}\right\} .
$$

Proof. At first, since $\hat{f}_{w}$ is the continuous extension of $f_{w}=\mathrm{id}_{K}$ for every $w$, we have $\hat{f}_{w}(\hat{A}) \subseteq \overline{f_{w}(A)}=\hat{A}_{w}$. Conversely, let $x \in \hat{f}_{w}^{-1}\left(\hat{A}_{w}\right)$ for every $w \in \Omega_{0}$. Let $w_{i} \in \Omega_{0}, i=1, \ldots, n, \alpha_{i} \in G_{w_{i}}^{+}$. Then since $A$ is a dense subset in $\hat{A}_{w_{i}}$, for every $i, 1 \leqslant i \leqslant n$, we may find an element $y_{i}$ such that

$$
y_{i} \in A \cap\left(\hat{f}_{w_{i}}(x)+U_{\hat{w}_{i}, \alpha_{i}}\right) \text {. }
$$

Now, since $w_{i}$ are pairwise independent, $\left(\left(\alpha_{1}, \ldots, \alpha_{n}\right),\left(y_{1}, \ldots, y_{n}\right)\right)$ is concordant. Thus, there exists $a \in A$ such that

$$
w_{i}\left(a-y_{i}\right)>\alpha_{i}, \quad i=1, \ldots, n .
$$

Then using the identity $\tilde{w}_{i}=\hat{w}_{i} \cdot \hat{f}_{w_{i}}$ from the proof of Proposition 4 , we have 


$$
\begin{aligned}
\tilde{w}_{i}(a-x) & =\hat{w}_{i} \cdot \hat{f}_{w_{i}}(a-x)=\hat{w}_{i}\left(a-\hat{f}_{w_{i}}(x)\right) \\
& =\hat{w}_{i}\left(a-y_{i}+y_{i}-\hat{f}_{w_{i}}(x)\right)>\alpha_{i}, \quad 1 \leqslant i \leqslant n .
\end{aligned}
$$

Therefore, $A$ is a dense subset in $\cap\left\{\hat{f}_{w}^{-1}\left(\hat{A}_{w}\right): w \in \Omega_{0}\right\}$.

Proposition 10. Let $w \in \Omega$ be such that $R_{\tilde{w}}=\hat{A}_{[P(\tilde{w})]}$. Then $R_{\hat{w}}=\left(\hat{A}_{w}\right)_{P(\hat{w})}$. Conversely, if $\Omega$ satisfies the W.R.A.T. for $A$ and if $R_{\hat{w}}=\left(\hat{A}_{w}\right)_{P(\hat{w})}$, then

$$
R_{\tilde{w}}=\hat{A}[P(\tilde{w})], \quad M(\tilde{w})=[P(\tilde{w})] \hat{A}[P(\tilde{w})] .
$$

Proof. Let $R_{\tilde{w}}=\hat{A}_{[P(\tilde{w})]}$ and let $x \in R_{\hat{w}}$. Then for $y \in \hat{f}_{w}^{-1}(x)$ there exists $z \in \hat{A}-P(\tilde{w})$ such that $y \cdot z \in \hat{A}$. Hence, $x \cdot \hat{f}_{w}(z) \in \hat{A}_{w}$ and $\hat{f}_{w}(z) \in \hat{A}_{w}-$ $P(\hat{w})$. Thus, $x \in\left(\hat{A}_{w}\right)_{P(\hat{w})}$. The converse inclusion is trivial. Conversely, let $R_{\hat{w}}=\left(\hat{A}_{w}\right)_{P(\hat{w})}$. Let $\Omega_{0}$ be the family of representatives of the equivalence classes of $\equiv$ such that $w \in \Omega_{0}$. Let $x \in R_{\tilde{w}}$. Then there exists $y_{w} \in \hat{A}_{w}-$ $P(\hat{w})$ such that $y_{w} \cdot \hat{f}_{w}(x) \in \hat{A}_{w}$. Further, since $\hat{K}_{w^{\prime}}$ is the quotient field of a domain $\hat{A}_{w^{\prime}}$ for every $w^{\prime} \in \Omega_{0}$, there exist $y_{w^{\prime}} \in \hat{A}_{w^{\prime}}, w^{\prime} \in \Omega_{0}, w^{\prime} \neq w$, such that $y_{w^{\prime}} \cdot \hat{f}_{w^{\prime}}(x) \in \hat{A}_{w^{\prime}}$. Now, by Lemma 8 , there exists $y \in \hat{K}$ such that $\hat{f}_{w^{\prime}}(y)=y_{w^{\prime}}, w^{\prime} \in \Omega_{0}$. Then by Lemma $9, y \in \hat{A}$, and using the identity $\tilde{w}=\hat{w} \cdot \hat{f}_{w}, y \in \hat{A}-P(\tilde{w})$. Further,

$$
\hat{f}_{w^{\prime}}(y \cdot x)=y_{w^{\prime}} \cdot \hat{f}_{w^{\prime}}(x) \in \hat{A}_{w^{\prime}}, \quad w^{\prime} \in \Omega_{0},
$$

hence $y \cdot x \in \hat{A}$. Therefore, $x \in \hat{A}_{[\hat{A}-P(\tilde{w})]}$. The converse inclusion is trivial. Further, it is clear that $[P(\tilde{w})] \hat{A}_{[P(\tilde{w})]} \subseteq M(\tilde{w})$. Let $y \in M(\tilde{w}) \subseteq \hat{A}_{[P(\tilde{w})]}$. Then there exists $z \in \hat{A}-P(\tilde{w})$ such that $y \cdot z \in \hat{A}$ and it is easy to see that $y \cdot z \in P(\tilde{w})$. Thus, $[P(\tilde{w})] \hat{A}_{[P(\tilde{w})]}=M(\tilde{w})$.

Lemma 11. Let $\Omega$ satisfy the W.R.A.T. for A. Then $\hat{A}=\cap\left\{R_{\tilde{w}}: w \in \Omega_{0}\right\}$ if and only if $A$ is a dense subset in $\left(R_{w}, \mathcal{T}_{w} \mid R_{w}\right)$ for every $w \in \Omega_{0}$.

Proof. If $A$ is a dense subset in $R_{w}$, then $\hat{A}_{w}=R_{\hat{w}}$ and the rest follows by Lemma 9. Conversely, let $\hat{A}=\cap\left\{R_{\dot{w}}: w \in \Omega_{0}\right\}$. If $A$ is not a dense subset in $R_{w}$ for some $w \in \Omega_{0}$, then $\hat{A}_{w} \neq R_{\hat{w}}$, and there exists $x_{w} \in R_{\tilde{w}}$ such that $\hat{f}_{w}\left(x_{w}\right) \notin \hat{A}_{w}$. Further, let for every $w^{\prime} \in \Omega_{0}, w^{\prime} \neq w, x_{w^{\prime}} \in R_{\tilde{w}^{\prime}}$. By Lemma 8 , there exists $x \in \hat{K}$ such that $\hat{f}_{w^{\prime}}(x)=x_{w^{\prime}}$ for every $w^{\prime} \in \Omega_{0}$. Thus, $x \in$ $\hat{f}_{w^{\prime}}^{-1}\left(R_{\left.\hat{w}^{\prime}\right)}=R_{\tilde{w}^{\prime}}, w^{\prime} \in \Omega_{0}\right.$. Hence, $x \in \hat{A}$ and $x_{w} \in \hat{A}_{w}$, a contradiction.

THEOREM 12. Let $A$ be a Prüfer domain such that there exists a family $\Omega_{0}$ of representatives of the equivalence classes of $\equiv$ such that $A$ is a dense subset in $\left(R_{w}, \sigma_{w} \mid R_{w}\right)$ for every $w \in \Omega_{0}$. Then $\hat{A}$ is a Prüfer ring.

Proof. By Lemma $11, \hat{A}=\cap\left\{R_{\tilde{w}}: w \in \Omega_{0}\right\}$. Let $a, b \in \hat{A}$ be such that the ideal $(a, b)$ in $\hat{A}$ is regular. Since $R_{\hat{w}}$ is a valuation domain, for every $w \in \Omega_{0}$ there exists $x_{w} \in R_{\hat{w}}$ such that $\left(\hat{f}_{w}(a), \hat{f}_{w}(b)\right) R_{\hat{w}}=x_{w} R_{\hat{w}}$. By Lemma 8 , there exists $x \in \hat{K}$ such that $\hat{f}_{w}(x)=x_{w}$ for every $w \in \Omega_{0}$. Hence $x \in R_{\dot{w}}$ for every $w \in \Omega_{0}$ and $x \in \hat{A}$. Then using the identity $\mathrm{pr}_{w}=\hat{f}_{w} \cdot \varphi$ from the proof of Lemma 8 , we obtain $(a, b)=x \hat{A}$, where $x$ is regular. Thus $(a, b)$ is invertible and, by the definition, $\hat{A}$ is a Prüfer ring. 
It should be observed that there is a reasonably large class of Prüfer domains which satisfy the conditions of Theorem 12 . In fact, every $h$-local Prüfer domain is of such type. Recall that an integral domain $A$ is said to be $h$-local if every nonzero ideal of $A$ is contained in only a finite number of maximal ideals, and if every nonzero prime ideal of $A$ is contained in only one maximal ideal. Then by [9, Theorem 22], $A$ is $h$-local if and only if $\hat{A} \cong \Pi\left\{\hat{A}_{M}: M \in \operatorname{mspec} A\right\}$, where mspec $A$ is the set of maximal ideals of $A, \hat{A}$ is the completion of $A$ in the $A$-topology, and $\hat{A}_{M}$ is the completion of $A_{M}$ in the $A_{M}$-topology, where for any ring $R$ the $R$-topology is the topology on $R$ with the ideals $r R, r \in R^{*}$, being a subbase for the open neighbourhoods of 0 in $R$.

The following lemma holds.

Lemma 13. Let $A$ be a h-local Prüfer domain. Then the topology $\sup \left\{\mathcal{G}_{w}\right.$ : $w \in \Omega\}$ on $A$ is the same as the A-topology.

Proof. Let $w_{i} \in \Omega, \alpha_{i} \in G_{w_{i}}^{+}$. Then there exist $a_{i} \in A, i=1, \ldots, n$, such that $w_{i}\left(a_{i}\right)>\alpha_{i}$. Then

$$
a_{1} A \cap \cdots \cap a_{n} A \subseteq \bigcap_{i=1}^{n} U_{w_{i}, \alpha_{i}} .
$$

Conversely, let $a \in A^{*}$. Then since $A$ is $h$-local, there exist only a finite number of maximal ideals $M_{1}, \ldots, M_{n} \in \operatorname{mspec} A$ such that $a \in \dot{M}_{i}$. Let $w_{i} \in \Omega, i=1, \ldots, n$, be such that $M_{i}=P\left(w_{i}\right)$, and we set $\alpha_{i}=w_{i}(a)$. Let $x \in A \cap \cap_{i=1}^{n} U_{w_{i}, \alpha_{i}}$. If $x \notin a A$, then there exists $w \in \Omega$ such that $w\left(x a^{-1}\right)$ $<0$. Since $w \neq w_{i}$, we have $a \notin P(w)$ and $w\left(x a^{-1}\right)=w(x) \geqslant 0$, a contradiction. Thus, $\cap_{i=1}^{n} U_{w_{i}, \alpha_{i}} \cap A \subseteq a A$ and $\sup \left\{\mathcal{F}_{w}\right\}$ on $A$ is the $A$-topology.

Since for every $h$-local Prüfer domain $A$, the family $\Omega_{0}=\{w \in \Omega: P(w)$ $\in \operatorname{mspec} A\}$ is a set of representatives of the equivalence classes of $\equiv, A$ satisfies the conditions of Theorem 12 and $\hat{A}$ is a Prüfer ring.

Further, we say that a ring $A$ with the total quotient ring $K$ is a $\mathscr{F}$-Prüfer ring, where $\mathscr{F}$ is a ring topology on $K$ if, for every maximal regular ideal $\mathrm{m}$ of $A,\left(A_{[\mathrm{m}]},[\mathrm{m}] A_{[\mathrm{m}]}\right)$ is a valuation pair associated with a valuation $w$ on $K$ such that $w$ is continuous in $\mathscr{F}$.

To generalize the definition of $w$-closed ideals, we say that an ideal $Q$ of $A$ is $\tilde{w} / \hat{A}$-closed for some $w \in \Omega$ if $x \in Q, y \in A$, and $\tilde{w}(x) \leqslant \tilde{w}(y)$ imply $y \in Q$.

We are now ready to prove the main theorem.

TheOREM 14. Let $A$ be a Prüfer domain. Then the following conditions are equivalent.

(1) $\hat{A}$ is $a \hat{\tilde{T}}$-Prüfer ring.

(2) For every maximal regular ideal $m$ of $\hat{A}$ there exists $w \in \Omega$ such that $\mathfrak{m}$ is $\tilde{w} / \hat{A}$-closed and $R_{\hat{w}}=\left(\hat{A}_{w}\right)_{P(\hat{w})}$ for every $w \in \Omega$.

(3) Every maximal regular ideal of $\hat{A}$ is open in $\hat{A}$ and $R_{\hat{w}}=\left(\hat{A}_{w}\right)_{P(\hat{w})}$ for every $w \in \Omega$. 
Proof. $(1) \Rightarrow(2)$. Let $m$ be a maximal regular ideal of $\hat{A}$. Then $\left(\hat{A}_{[\mathrm{m}]},[\mathrm{m}] \hat{A}_{[\mathrm{m}]}\right)$ is a valuation pair associated with a valuation $v$ continuous in $\hat{\sigma}$. Let $w=v \mid K$. It is easy to see that $w$ is a valuation on $K$. If $w$ is the trivial one, it follows since $w$ is continuous in $\mathcal{T}$, that $\mathcal{T}$ is discrete. Thus, $\hat{K}=K$, $\hat{A}=A, \mathfrak{m}=M(w) \cap A=(0)$, a contradiction with $\mathfrak{m}$ regular. Hence $w$ is nontrivial, $w \in \Omega, \tilde{w}$ and $v$ are the continuous extensions of $w$ on $\hat{K}$. Since $\hat{K}$ is Hausdorff, we obtain $\tilde{w}=v$. Hence $R_{\tilde{w}}=\hat{A}_{[\mathrm{m}]}, M(\tilde{w})=[\mathrm{m}] \hat{A}_{[\mathrm{m}]}$, and it is easy to see that $\hat{A}_{[\mathrm{m}]}=\hat{A}_{[P(\tilde{w})]}, P(\tilde{w})=\mathrm{m}$. Thus, $\mathrm{m}$ is $\tilde{w} / \hat{A}$-closed and the rest follows by Proposition 10 .

(2) $\Rightarrow$ (3). Let $m$ be a maximal regular ideal of $\hat{A}$ and let $x \in \mathfrak{m}$ be regular. Then for $z \in U_{\tilde{w}, \tilde{w}(\mathbf{x})} \cap \hat{A}$ we have $\tilde{w}(y+z) \geqslant \tilde{w}(z) \geqslant \tilde{w}(x)$ if $\tilde{w}(y) \geqslant \tilde{w}(z)$ and $\tilde{w}(y+z)=\tilde{w}(y)$ if $\tilde{w}(y)<\tilde{w}(z)$. Hence $y+z \in \mathfrak{m}$, and $\mathfrak{m}$ is open in $\hat{A}$.

(3) $\Rightarrow(1)$. Let $\mathrm{m}$ be a maximal regular ideal of $\hat{A}$ and $P=A \cap \mathrm{m}$. Since $A$ is a dense subset in $\hat{A}$, and $\mathrm{m}$ is open in $\hat{A}$, it follows that $\mathrm{m}=\overline{\mathrm{m} \cap A}=\bar{P}$, where the vinculum denotes the closure in $\hat{A}$. Since $\mathrm{m}$ is regular, we have $P \neq(0)$. Let $w \in \Omega$ be such that $R_{w}=A_{P}$. By Proposition $10, R_{\tilde{w}}=\hat{A}_{[P(\tilde{w})]}$. It is clear that $\bar{P} \subseteq P(\tilde{w})$. Suppose that there exists $x \in P(\tilde{w})-\bar{P}$; then for some $w_{1}, \ldots, w_{n} \in \Omega, \alpha_{i} \in G_{w_{i}}^{+}, i=1, \ldots, n$, we have

$$
\left(x+\bigcap_{i=1}^{n} U_{\tilde{w}_{i}, \alpha_{i}}\right) \cap P=\varnothing .
$$

On the other hand, there exists $a \in A$ such that

$$
\tilde{w}_{i}(a-x)>\alpha_{i}, \quad i=1, \ldots, n .
$$

Again, there is no loss of generality in assuming that $w \in\left\{w_{1}, \ldots, w_{n}\right\}$, say $w=w_{1}$. Then since $P=P\left(w_{1}\right)$, it follows that

$$
\alpha_{1}<\tilde{w}_{1}(a-x)=\min \left\{w_{1}(a), \tilde{w}_{1}(x)\right\}=0,
$$

a contradiction. Thus, $P(\tilde{w})=\bar{P}=\mathrm{m}$, and $\left(\hat{A}_{[\mathrm{m}]},[\mathrm{m}] \hat{A}_{[\mathrm{m}]}\right)$ is a valuation pair associated with the valuation $\tilde{w}$ continuous in $\hat{\mathcal{T}}$. Therefore, $\hat{A}$ is a $\hat{\mathcal{T}}$-Prüfer ring.

We have not been able to show that there exists a Prüfer domain $A$ such that $\hat{A}$ is not a $\hat{\mathcal{J}}$-Prüfer ring, and we do not know if there is a Prüfer domain $A$ such that $\hat{A}$ is a Prüfer ring but $\hat{A}$ is not a $\hat{\mathcal{T}}$-Prüfer ring.

Finally, we say that a ring $A$ with the total quotient ring $K$ is a $\mathscr{F}-N$-ring for some ring topology $\mathscr{F}$ on $K$, if for every maximal regular ideal $\mathrm{m}$ of $A$, $\left(A_{[\mathrm{m}]},[\mathrm{m}] A_{[\mathrm{m}]}\right)$ is a valuation pair associated with a valuation $w$ on $K$ continuous in $\mathcal{F}$ and such that $G_{w} \cong Z$.

THEOREM 15. Let $A$ be an almost Dedekind domain. Then the following conditions are equivalent.

(1) $\hat{A}$ is $a \hat{\tilde{J}}$-N-ring.

(2) For every maximal regular ideal $\mathfrak{m}$ of $\hat{A}$ there exists $w \in \Omega$ such that $\mathfrak{m}$ is $\tilde{w} / \hat{A}$-closed.

(3) Every maximal regular ideal of $\hat{A}$ is open in $\hat{A}$. 
Proof. The proof of implications $(1) \Rightarrow(2) \Rightarrow(3)$ is quite the same as the one of Theorem 14.

(3) $\Rightarrow(1)$. Let $\mathfrak{m}$ be a maximal regular ideal of $\hat{A}$ and $P=\mathfrak{m} \cap A$. Again, $\mathfrak{m}=\overline{\mathfrak{m} \cap A}=\bar{P}$, and it follows that $P \neq(0)$. Since $A$ is almost Dedekind, $A_{P}=R_{w}$ is a discrete rank one valuation domain and $P$ is a maximal ideal of $A$. Then by Proposition 6, $R_{\hat{w}}=\hat{A}_{w}$ and by Proposition $10, R_{\bar{w}}=\hat{A}_{[P(\tilde{w})]}$. The rest of this proof is the same as the one of Theorem 14.

\section{REFERENCES}

1. M. B. Boisen and M. D. Larsen, Prüfer and valuation rings with zero divisors, Pacific J. Math. 40 (1972), 7-12. MR 46 \#9025.

2. N. Bourbaki, General topology. Parts 2, 3, Hermann, Paris; Addison-Wesley, Reading, Mass., 1966. MR 34 \# 5044.

3. M. Fukawa, On the theory of valuations, J. Fac. Sci. Univ. Tokyo Sect. I 12 (1965), 57-79. MR 32 \#4126.

4. M. Griffin, Prüfer rings with zero divisors, J. Reine Angew. Math. 239/240 (1969), 55-67. MR 41 \# 188.

5. __ Rings of Krull type, J. Reine Angew. Math. 229 (1968), 1-27. MR 36 \#3778.

6. P. H. Kelly and M. D. Larsen, Valuation rings with zero divisors, Proc. Amer. Math. Soc. 30 (1971), 426-430. MR 44 \#2745.

7. M. D. Larsen, $A$ generalization of almost Dedekind domains, J. Reine Angew. Math. 245 (1970), 119-123. MR 42 \#7662.

8. M. E. Manis, Extension of valuation theory, Bull. Amer. Math. Soc. 73 (1967), 735-736. MR 36 \# 1436.

9. E. Matlis, Torsion-free modules, Univ. of Chicago Press, Chicago, Ill., 1972.

10. J. L. Mott, Convex directed subgroups of a group of divisibility, Canad. J. Math. 26 (1974), 532-542. MR 51 \#468.

Department of Mathematics, Mining University, 70833 Ostrave 4, Czechoslovakia 